\title{
Teaching Inclusive Thinking to Undergraduate Students in Computing Programs
}

\author{
Stephanie Ludi \\ Dept. of Computer Science \& \\ Engineering \\ University of North Texas, USA \\ Stephanie.ludi@unt.edu
}

\author{
Matt Huenerfauth \\ Department of Information Sciences \& \\ Technologies \\ Rochester Institute of Technology, USA \\ matt.huenerfauth@rit.edu
}

\author{
Vicki Hanson \\ Dept. of Information Sciences \& \\ Technologies \\ Rochester Institute of Technology, USA \\ vlhics@rit.edu
}

\author{
Nidhi Rajendra Palan \\ Department of Information Sciences \& \\ Technologies \\ Rochester Institute of Technology, USA \\ nxp4195@rit.edu
}

\begin{abstract}
An increasing importance of accessibility awareness and knowledge emanates from a moral imperative and as an employment differentiator. It is important that educational programs have a demonstrated ability to teach these skills. In this paper, we focus on the role that educational courses can play in increasing accessibility awareness for undergraduate students. We review literature indicating that a number of accessibility teaching interventions have been reported; yet the evaluation of their effectiveness has not been conducted in a consistent manner. We report on our 3-semester evaluation of undergraduate students' accessibility awareness and knowledge following a week of accessibility lectures as part of courses on Human-Computer Interaction (HCI), where a subset of students also interact with stakeholders with disabilities during the conduct of the course projects. Gains in awareness and knowledge occur when accessibility lectures were part of the course. These gains are compared across the teams who interacted with a person with a disability and teams with no such interaction. In addition, we provide the test battery developed to measure these skills, to enable other researchers to conduct evaluations of the effectiveness of interventions for teaching inclusive thinking in undergraduate computing at their own institutions
\end{abstract}

\section{ACM Reference Format}

Stephanie Ludi, Matt Huenerfauth, Vicki Hanson, Nidhi Rajendra Palan, and Paula Conn. 2018. Teaching Inclusive Thinking to Undergraduate Students in Computing Programs. In SIGCSE '18: 49th ACM Technical Symposium on Computing Science Education, Feb. 21-24, 2018, Baltimore, MD, USA. ACM, NY, NY, USA, 6 pages. https://doi.org/10.1145/3159450.3159512

Permission to make digital or hard copies of all or part of this work for personal or classroom use is granted without fee provided that copies are not made or distributed for profit or commercial advantage and that copies bear this notice and the full citation on the first page. Copyrights for components of this work owned by others than ACM must be honored. Abstracting with credit is permitted. To copy otherwise, or republish, to post on servers or to redistribute to lists, requires prior specific permission and/or a fee. Request permissions from Permissions@acm.org.

SIGCSE '18, February 21-24, 2018, Baltimore, MD, USA

(C) 2018 Association for Computing Machinery.

ACM ISBN 978-1-4503-5103-4/18/02...\$15.00

https://doi.org/10.1145/3159450.3159512

\author{
Paula Conn \\ Department of Computing and Information \\ Sciences \\ Rochester Institute of Technology, USA \\ pxg5962@rit.edu
}

\section{INTRODUCTION}

A statement from the current draft of the 2018 ACM Code of Ethics and Professional Conduct [1] inspired us, as accessibility researchers and educators, to think whether, in fact, we were educating our computing students in a way that will instill in them the notion of inclusive thinking as a critical aspect of all design and development. Specifically, the code states:

"Inequities between different groups of people may result from the use or misuse of information and technology. Technologies should be as inclusive and accessible as possible. Failure to design for inclusiveness and accessibility may constitute unfair discrimination."

We became interested in whether the training we provide as university computing instructors would influence students as future technology developers to design systems, software, and services to be usable by people with diverse abilities. In section 4.2 , we share excerpts from documents submitted by students about software design projects; one of these quotations includes:

"[Our site] does not account for disabilities. Users must accommodate for their disabilities prior to using the site."

Certainly, this quotation does not reflect the ideals expressed in the ACM code. In this paper, we report on a study that investigates the efficacy of two approaches to teaching inclusive thinking to address this research question: To what extent do these interventions lead to increased student perceptions of the ethical role of computing in addressing accessibility and inclusion?

- Undergraduate computing students participate in a required course on human-computer interaction, with a week of lectures on accessibility and a semester-long team project to design software or a website.

- $\quad$ Same as above, with some first-hand personal interaction during the semester with a person with a disability during the design or evaluation of the team project.

We highlight two specific contributions of this paper. The first is in evaluation of whether any of the interventions on accessibility (lectures or interaction with a person with a disability) measurably increase students' awareness and / or knowledge of accessibility. 
The second is making available our testing instruments for use by other researchers.

This paper is organized as follows. First, we review the context for curriculum development that includes accessibility as an essential component of the ethical and practical training of computing professionals. Next, we describe the methods being employed in our research and our research findings. We conclude with the context for this work from our ongoing research on undergraduate teaching of accessibility.

\section{RELATED RESEARCH}

The importance of teaching accessible design practices at the undergraduate level has been argued both on moral and practical reasons. There is a growing awareness among employers of the need to hire designers and developers knowledgeable about issues of accessibility and inclusion $[9,10,22]$; this may be due to the growing number of worldwide standards and regulations governing accessible technologies, the corresponding increase in demand for skills to comply with these regulations, and the need to address ethics and social responsibility in technology innovation. In a survey of 900 web accessibility practitioners, over $82 \%$ indicated ethical reasons ("It's the right thing to do") as a personal motivation for accessible design [24].

Despite such calls for more accessibility-aware developers, even recent publications have noted the lack of principled methods for teaching accessibility $[12,18]$. A number of approaches have been reported anecdotally, but there have been few attempts to measure outcomes. We consider here a variety of interventions that have been used to teach accessibility principles (see $[12,18]$ for further consideration of approaches).

Most commonly, degree programs include accessibility content in upper-level undergraduate courses on accessibility, or in courses on related topics, such as human-computer interaction (HCI) [3, 13, 14, 18, 20]. Notably, the 2013 ACM/IEEE-CS Joint Task Force on Computing Curricula includes accessibility topics within HCI [4]. The types of educational interventions in prior computingcurriculum efforts on accessibility and inclusion can be classified into broad categories:

- Lectures: Lectures on accessibility can be included in a computing class or guest speakers with experience in accessible design may visit classes or departments, e.g. [23]. Some courses enable students to experience firsthand how users with disabilities access computers or the web [3].

- Projects: Authors also describe courses with student programming or design projects, sometimes in teams, with the topic related to people with disabilities. Some faculty gather project topics from clients, including local disability service organizations that have in mind a specific need for a user with a disability $[2,15]$. In other cases, teams may select an accessibility-related topic on their own [23], often after exposure to such topics in prior courses.

- Direct interaction with target users: In some courses, individuals with disabilities may visit classes to discuss their first-hand experiences with technology, e.g. [23]. Students conducting projects sometimes interact directly with people with disabilities during the requirements gathering or evaluation stage of the projects $[13,14,15]$.
- Collaborating with a team member with a disability: As students with disabilities enroll in computing degree programs, more students have personal experience working with a team member with a disability on a project [9]. For example, Huenerfauth [7] has organized research projects with significant numbers of deaf and hard-of-hearing students collaborating with hearing students.

These interventions typically happen within an individual course. There are some reports, however, in which accessibility is considered throughout an undergraduate curriculum [19, 23], and weaving accessibility throughout a degree may enhance students' perception that the topic is integral to the computing profession. Similar in conception was the work of Shinohara, Bennett, and Wobbrock [20] in which a User-Centered Design course was taught with the expectation that accessible design was part of the overall process, not "someone else's job."

While many prior publications on educational interventions for accessibility describe faculty and student experiences, to our knowledge none performed comparisons of alternative methods to measure efficacy. We note a recent call by Lewthwaite and Sloan [12] to move beyond descriptions of teaching approaches to evaluate and inform methods of teaching accessibility.

In the work reported here, we address the need for evaluation of teaching accessibility. In line with Lewthwaite and Sloan [12], we consider accessibility instruction to cover the design and development of websites and software such that these would be usable by people with disabilities. We state in advance, however, that our research procedures do not test for specific accessibility skills. Rather, our methods examine attitudes and awareness regarding disability as part of inclusive thinking about technology design and evaluation.

\section{METHODS}

We report on 3 semesters of measuring efficacy for two accessibility interventions: accessibility lectures and lectures plus first-hand interaction with a person with a disability. We use a number of pre- and post-test survey measures to examine student changes in awareness and attitudes about accessibility and people with disabilities. In addition, students' documents submitted during the course were retained and qualitatively analyzed to examine the degree of inclusive thinking. This research was conducted at the Rochester Institute of Technology (RIT), a private university in the United States. Approval was obtained from the university's Internal Review Board.

\subsection{Research participants}

The study was conducted as part of required courses in Information Technology (IT) and Software Engineering (SE). At RIT students in Years 2 and 3, majoring in IT or SE, respectively, are required to take a course in HCI (a separate course within each major). The courses may be taken later by students. We report here on three 16-week semesters of these HCI classes (9 classes in total). During the first semester of the study, one of the authors served as the instructor for one course.

Students were assigned to one of two groups:

- NoExposure: The HCI courses for both majors contain a semester-long project, in which teams of 4-5 students design a 
mobile app, website, or desktop application while following a design process and submitting reports on their progress. There were 35 NoExposure student teams (20 SE, 15 IT). (These materials were qualitatively analyzed; see section 4.2.) Both groups received approximately a week of lectures about accessibility, with content on: (1) diversity of human sensory abilities, including experiences of people with common visual impairments and who are Deaf/deaf or hard-of-hearing; (2) human motor system and various movement impairments; (3) diversity in human cognitive or learning abilities; (4) common assistive technologies for accessing computers (e.g. screen readers, magnification, alternative input); (5) U.S. legal requirements for technology accessibility; (6) key aspects of website design for accessibility (e.g. alt text, captioned media). The slides for these lectures are available on our laboratory website. $^{1}$

- Exposure: The second group experienced the same as above, plus they also had first-hand interaction with someone with a disability during the semester in the context of the design or evaluation of their team project. Teams met with someone to receive requirements or receive feedback on a prototype design, and in some cases, this person was a member of their team (a fellow student). There were 14 Exposure teams (5 SE, 9 IT).

\subsection{Survey Measurement Instruments}

A SurveyMonkey survey was created that included the three measurement instruments listed below (in that order). Students in participating classes were asked to complete the pre-test during the first 2 weeks of the semester. They were asked to complete the post-test (the same survey) during the last two weeks of the 16week semester. In one IT class, students completed this battery during class time. In other classes, they were emailed the survey link from the instructor and asked to complete the survey at home. This email recruitment resulted in few students taking the survey. This recruitment procedure, therefore, was changed after the 1st semester to offer a $\$ 100$ Amazon gift certificate raffle among students who completed the survey.

\subsubsection{Scenario}

First, participants were presented with the voting-machine problem scenario from Ludi [13], where students are asked to design a voting kiosk for an election. They were then asked two open-ended questions: The first asked them to discuss key points that they would keep in mind in the design of the voting system. The second question asked which potential voters they would include when testing their prototype. The scenario did not mention disabilities. The two questions were designed to be unbiased/neutral, so any mentions of accessibility by the students were based on their awareness of accessibility importance in the design and evaluation of software.

\subsubsection{Interaction with Disabled Persons (IDP) scale}

IDP is a standardized 20-item instrument that assesses both desirable and undesirable emotions experienced when interacting

\footnotetext{
${ }^{1}$ http://cair.rit.edu/cse2018
}

with people with disabilities [5, 6, 21]. The scale consists of 20 statements about interacting with people with disabilities. For each statement, respondents are asked to indicate the extent to which they agree with the statement using a 6-point Likert scale. In factor-analysis work, the 20 item IDP scale has been found to best load onto six factors describing both positive and negative reactions when interacting with people with disabilities [5], listed below with an example IDP item from each subscale. Of the 20 question items, three items had been found not to be relevant to the factors below [5] and, therefore, are not included in the analyses presented in 4.1.1.

- Discomfort meeting someone with a disability: "I am afraid to look at the person straight in the face."

- Sympathy: "It is rewarding when I am able to help."

- Uncertainty: "I feel uncomfortable and find it hard to relax."

- Fear: "I am grateful that I do not have such a burden."

- Coping relates to the view a person takes towards someone with a disability: "After frequent contact, I find I just notice the person not the disability."

- Vulnerability: "Contact with a disabled person reminds me of my own vulnerability."

\subsubsection{Accessibility Survey}

Several researchers have surveyed professionals about their awareness of the issue of accessibility, especially in regard to websites, e.g., [11]. No instrument was directly applicable to our needs, however, due to differences in regulations mentioned in the questions (some of which are now out-of-date), or questions relating to the participant's current employer (our participants are students), etc. We, therefore, extracted relevant questions from prior surveys into our own survey.

This survey first asked about accessibility awareness. Participants were asked to indicate whether they had knowledge of or personal experience with people with specific disabilities (low vision, blindness, hearing loss, autism, learning disabilities, intellectual disabilities, motor disabilities, or older people). For these 8 items, radio button options were used to indicate either a) knowledge of or b) personal experience with each case.

Second, there were a number of questions about accessibility knowledge in design and development. To begin, participants were asked 8 questions regarding whether they had read about or had done website development for people with disabilities. Next, they were asked 10 questions regarding their familiarity or experience with specific web-accessibility issues (e.g., CSS, alternative text, colors). There was a further set of 6 questions regarding their familiarity or experience with several software accessibility issues (e.g., screen-reader interfaces, avoiding responses that require a fixed time limit, etc.). The final two items were Yes / No questions asking whether students had previously been involved in the design/development of websites or software and, if yes, whether accessibility had been taken into account.

\subsubsection{Relation to Our Interventions and Availability}

Our survey questions about the scenario and accessibility knowledge are related to the in-class lecture content; our survey questions about accessibility awareness and the IDP require an understanding or empathy about disability that could influence 
requirements gathering. Our full survey, thus, addresses questions both about knowledge gained regarding specific taught topics, as well as attitudinal changes that might result from accessibility instruction. The measures of accessibility awareness and accessibility knowledge are broad categories, each with multiple aspects. We believe these categories are useful in considering whether varied teaching interventions promote an understanding of the technical aspects of accessibility and / or whether they promote an awareness of the need to consider accessibility requirements during development.

Our complete survey, including the scenario [13], IDP [5], accessibility awareness, and accessibility knowledge instruments can be downloaded from our lab website. ${ }^{1}$

\subsection{Qualitative Coding of Student's Documents}

We collected 236 student-submitted documents during the three semesters of evaluation. There were more NoExposure student teams $(n=35)$ than Exposure teams $(n=14)$. Given this, there were more documents from NoExposure teams (134 documents, 220,081 words) than from Exposure teams (38 documents, 50,677 words). Image (35 NoExposure, 16 Exposure) and video (11 NoExposure, 2 Exposure) submissions were also collected.

As part of a qualitative analysis, two researchers independently coded all student materials to identify themes related to students considering accessibility or people with disabilities. All thematic codes were developed following the methods of Saldana [26]. Student teams submitted multiple assignments on the same project, occasionally with redundant information. Repeated assignment content was coded once per team. Once each researcher completed their individual coding for each semester's data, they reconvened and recoded any missing quotes, before comparing the results for agreement.

While the data was labeled with multiple thematic codes during this analysis, here we focus on two: (1) A team noted an accessibility barrier but chose not to address it, and (2) Students referenced to accessibility guidelines, e.g. WCAG. A total of 19 instances of these two thematic codes were labeled. To calculate inter-rater reliability (IRR), we followed the recommendation of [27] for qualitative research involving thematic coding of transcript-type data using a large set of thematic codes (thereby reducing the likelihood of chance agreement) and when individual text excerpts may be labeled by more than one code. To unitize our data for IRR analysis, we assembled all text excerpts for which either annotator had assigned one of these two codes and then calculated proportional agreement (percentage of excerpts in the union set for which both annotators assigned identical codes), obtaining an IRR of $85 \%$.

\section{RESULTS AND DISCUSSION \\ 4.1 Quantitative Findings from Surveys}

In our three semesters of testing, 95 students completed both the pre- and post-test. Of these students, 63 were in the NoExposure group, and 32 were in the Exposure group (described in section 3.1). Our quantitative analyses reported below were conducted only on the sample who had completed both the pre- and post-test for each measure. Results are presented separately below, for each portion of the survey: the scenario, the IDP scale, and accessibility survey (described in section 3.2). For each measure, we conducted the following analyses:

- Comparing NoExposure and Exposure: To determine whether firsthand exposure to people with disabilities led to differences in each measure, we first calculated a 'change' score for each student by subtracting their pre-test score from their post-test score. We then compared these 'change' values for the two groups using an unpaired Mann Whitney U-test. This test would reveal any significant differences between students who did or did not have firsthand exposure to someone with a disability.

- Comparing Pre- and Post- Scores for All Students: For all students (NoExposure and Exposure combined), we performed a Wilcoxon Signed-Rank test to determine whether there was a significant difference between their preand their post- values. These tests would reveal significant changes between the beginning and the end of the semester. If a significant difference had been observed in the "Comparing NoExposure and Exposure" test above, then this test was performed separately for each group.

\subsubsection{Scenario}

We use Ludi's [13] rubric to measure the extent to which students considered accessibility in the voting scenario; a total of 93 students completed both the pre- and post- test for this measure. Participants' responses to each of the two open-ended scenario questions (see section 3.2.1) received a binary score indicating whether or not their response mentioned accessibility. For both pre- and post-testing, each student could have a score of 0,1 , or 2 , depending on whether or not they had mentioned accessibility in response to neither, one, or both questions.

Comparing NoExposure and Exposure: No significant difference was observed in the change in scenario scores between the two groups ( $U=889, Z=0.58, p=0.56$, two-tailed).

Comparing Pre- and Post- Scores for All Students: We observed a significant difference in the pre- and post- scores $(W=$ $736, Z=3.66, p<.001$, two-tailed). There were a greater number of accessibility mentions in the responses at the end of the semester than in the responses at the beginning of the semester.

Using this scenario, Ludi [13] had originally examined differences in inclusive thinking about software design and evaluation; in that study, significant differences in considering accessibility were found between SE students who did and did not meet people with disabilities as part of their team projects. In contrast, in the current study, when examining the change (pre- to post-) in students' responses, there were gains in this measure during the semester for students in both the Exposure and NoExposure groups.

\subsubsection{IDP Scale}

A total of 95 students completed both the pre- and post- survey for the IDP scale items.

Comparing NoExposure and Exposure: No significant difference between the two groups was observed for changes in scores for five of the subscales: Discomfort, Uncertainty, Fear, Copying, nor Vulnerability. However, for the Sympathy subscale, a significant difference was observed between the two groups $(U=$ $741, Z=2.1, p<0.05$, two-tailed). Median change in scores for each 
group (NoExposure $=0$, Exposure $=-0.25$ ); lower scores on this scale indicate greater degree of Sympathy.

Comparing Pre- and Post- Scores for All Students: No significant difference was observed between the pre- and postscores for five of the subscales: Discomfort, Uncertainty, Fear, Copying, nor Vulnerability. For the Sympathy subscale, because a significant difference had been observed in the "Comparing NoExposure and Exposure" test above, this pre- and postcomparison was performed separately for each group.

- NoExposure: We did not observe any significant difference in the Sympathy pre- and post- scores for the students who did not have firsthand exposure to people with disabilities $(W=$ 78, $Z=0.37, p=.71$, two-tailed).

- Exposure: We observed a significant difference in the preand post- scores ( $W=-170, Z=-2.42, p<.05$, two-tailed). For students who had firsthand interaction with someone with a disability, their scores were significantly lower at the end of the semester, which indicates a greater degree of Sympathy, with median values pre $=4.74$, post $=4.45$.

The IDP scale did not generally show shifts in students' attitudes about interacting with people with disabilities. Only the Sympathy factor showed gains in this analysis, and it is reasonable to see how firsthand interactions with people with disabilities during the semester may have led to changes in this subscale. We can speculate why no additional differences were observed: It may be the case that the IDP scale is not sensitive enough, given the numbers we have for participants. In earlier work with IDP, findings were based on over a thousand participants [6, 21]. Also, we have to consider that the IDP scale, developed in 1999, may be dated, reflecting language and attitudes not well-suited to the current United States culture.

\subsubsection{Accessibility Survey: Accessibility Awareness}

For accessibility awareness, each of the 8 items was scored as: 0 if the participant did not indicate either knowledge of or personal experience with a disability; 1 if they indicated knowledge of a disability; or 2 if they indicated personal experience with a disability. Thus, each student could receive a total score from $0-16$ for accessibility awareness. A total of 82 students completed the preand post- survey for this measure.

Comparing NoExposure and Exposure: No significant difference was observed in the change in scenario scores between the two groups ( $U=709, Z=0.86, p=0.39$, two-tailed).

Comparing Pre- and Post- Scores for All Students: We did not observe any significant difference in the pre- and post- scores ( $W=238, Z=0.92, p<.36$, two-tailed).

For this measure, we did not observe any differences in scores between the two groups or when comparing pre- and post-testing.

\subsubsection{Accessibility Survey: Accessibility Knowledge}

For accessibility knowledge, each of the 24 items was scored as follows: 0 if the participant did not indicate they were familiar with or had taken into account issues for accessible development; 1 if they indicated familiarity with issues in accessible development; or 2 if they indicated had taken into account issues in accessible development. The two final questions responses were scored as a 1 if students answered Yes to the question. In total, each student could receive a total score from $0-50$ for accessibility knowledge.
A total of 78 students completed both the pre- and post- survey for this measure.

Comparing NoExposure and Exposure: No significant difference was observed in the change in scenario scores between the two groups ( $U=806, Z=-0.79, p=0.43$, two-tailed).

Comparing Pre- and Post- Scores for All Students: We observed a significant difference in the pre- and post- scores ( $W=$ $1330, Z=3.58, p<.001$, two-tailed). The accessibility knowledge scores were significantly higher in the post-test (median $=35)$ than the pre-test (median $=30$ ).

In sum, we found significant gains in this measure when comparing the post- and pre- scores for all students. Students in both groups received a week of lectures on accessibility in their $\mathrm{HCI}$ course, and we found gains in self-reported knowledge about accessibility. This result helps validate efforts to teach accessibility, e.g., $[10,14,18,20,22]$, in line with calls to provide concrete evidence of effectiveness $[12,18]$.

\subsection{Qualitative Analysis of Student's Documents}

\subsubsection{Students Explicitly Discuss Accessibility Guidelines}

After coding 236 student team documents, we found that none of the teams with exposure to an individual with disabilities ( 0 out of 14) referenced existing accessibility guidelines (e.g., WCAG, W3C) in their project. Instead, we noticed that students tended to rely on the information gained from their personal interaction with someone with a disability. On the other hand, 4 out of 35 NoExposure teams referenced such guidelines: Three teams only considered guidelines relating to color choices (e.g. for colorblindness), and only one team considered a broader set of guidelines, including audio and text.

\subsubsection{Accessibility Considered but not Addressed}

In the NoExposure group, 5 of the 35 teams mentioned that they noticed an accessibility barrier but they decided not to address it. Reasons provided for why they did not address the barrier included: the students felt it was outside their project scope, time limitations prevented them from doing so, or the barrier had not been initially foreseen. For the 14 Exposure teams only one noted an accessibility barrier but did not address it.

Several teams put the onus on the user with a disability. One NoExposure team developed an application that could only be used by users without disabilities, stating that their project "does not account for disabilities. Users must accommodate for their disabilities prior to using the site." Another NoExposure team who developed an application for a mobile phone said that users had to be "physically and mentally capable of using the Android application and understanding the information that it provides." A third NoExposure team decided against implementing keyboard shortcuts that were originally included in their requirements document for accessibility reasons, explaining they "decided against keyboard shortcuts. Not very useful and would conflict with OS keyboard shortcuts." Of the remaining two NoExposure teams who noted accessibility barriers but chose not to address them, one said their "color scheme could use some tweaks to follow accessibility guidelines," and the other team noted that "alt text [could be added] to the portal." These quotations above are especially notable in light of the quantitative finding reported in 
section 4.1.2, where we found a significantly greater improvement in Sympathy IDP sub-scores for students in the Exposure group.

Only one Exposure team (who had firsthand interaction with an individual with a disability) noted an accessibility barrier yet chose not to address it. Their project required users to have the "ability to use a mobile device or have computer internet access" and specifically targeted taxi drivers who did not "have any disabilities that would affect their driving."

\section{CONCLUSIONS AND FUTURE WORK}

This study compared two interventions for promoting inclusive thinking about accessibility and people with disabilities among undergraduate computing students participating in required courses on human-computer interaction. One intervention involved a week of lectures about accessibility; the other intervention included these lectures plus first-hand interaction with someone with a disability during the semester, in the context of a team design project.

Based on our analysis with multiple pre- and post-test survey measurements, for both interventions we observed post-test gains on three measures: (1) more mentions of accessibility on the voting-machine design scenario, (2) greater Sympathy attitudinal scores on the IDP scale, and (3) increased self-reported accessibility knowledge. To our knowledge, this study is the first such quantitative evidence of these changes after accessibility-focused educational interventions.

When comparing the two interventions in this study, we found significant gains in the Sympathy scores only for those students in the Exposure group. Our qualitative analysis of student's work shed additional light on this finding: Specifically, while students in both groups experienced increased accessibility knowledge (leading them to notice circumstances when accessibility barriers may arise in their projects), this knowledge may not be enough to motivate students to address accessibility barriers. Our findings suggest that interventions that lead to firsthand interaction or increased empathy with technology users with disabilities may help students go from noticing a barrier to taking steps to address it.

The current work reports on two teaching interventions during three semesters of HCI classes. In future work, we will also collect survey responses and perform qualitative analysis of submitted project documents from students two years after the HCI course was concluded. This future work will examine lasting impact from these interventions on students' accessibility knowledge or the degree to which they are motivated to consider people with disabilities when designing software or technology. We will use our current findings to inform our deeper qualitative analysis of the materials collected from students in these courses to understand how to most effectively promote inclusive design thinking among these future computing professionals.

\section{ACKNOWLEDGMENTS}

This work is supported by the National Science Foundation, Award 1540396.

\section{REFERENCES}

[1] ACM Ethics. Retrieved April 28, 2017 from https://ethics.acm.org/
[2] Buckley, M., Kershner, H., Schindler, K., Alphonce, C., Braswell, J. 2004. Benefits of using socially-relevant projects in computer science and engineering education. In Proceedings of the 35th SIGCSE Technical Symposium on Computer Science Education, ACM, New York, NY, 482-486.

[3] Jim A. Carter and David W. Fourney. 2007. Techniques to assist in developing accessibility engineers. In Proceedings of the 9th international ACM SIGACCESS conference on Computers and accessibility. ACM, NY, 123-130.

[4] Computer Science Curriculum 2013: Curriculum Guidelines for Undergraduate Degree Programs in Computer Science. 2013. Retrieved 20 April, 2017 from https://www.acm.org/education/CS2013-final-report.pdf

[5] Chris Forlin, Gerard Fogarty, \& Annemaree Caroll. 1999. Validation of the factor structure of the Interactions with Disabled Persons Scale. Australian J. of Psychology, 51(1), 50-55.

[6] Lindsay Gething and Barbara Wheeler. 1992. The Interaction with Disabled Persons scale: A new Australian instrument to measure attitudes toward people with disabilities. Australian J. of Psychology, 44 (22), 75-82.

[7] Matt Huenerfauth. 2010. Participation of high school and undergraduate students who are deaf in research on American Sign Language animation. SIGACCESS Access. Comput. 97 (June 2010), 14-24.

[8] Maurits Kaptein and Judy Robertson. 2012. Rethinking statistical analysis methods for $\mathrm{CHI}$. In Proceedings of the SIGCHI Conference on Human Factors in Computing Systems (CHI '12). ACM, NY, USA, 1105-1114.

[9] Andrew J. Ko and Richard E. Ladner. 2016. AccessComputing Promotes Teaching Accessibility. ACM Inroads 7, 4 (November 2016), 65-68.

[10] Richard E. Ladner and Matt May. 2017. Teaching Accessibility. In Proceedings of the 2017 ACM SIGCSE Technical Symposium on Computer Science Education (SIGCSE '17). ACM, New York, NY, USA, 691-692.

[11] Jonathan Lazar, Alfreda Dudley-Sponaugle, Kisha-Dawn Greenidge. 2004. Improving Web Accessibility: A Study of Webmaster Perceptions. Computers and Human Behavior, 20(2), 269-288.

[12] Sarah Lewthwaite and David Sloan. 2016. Exploring pedagogical culture for accessibility education in computing science. In Proceedings of the 13th Web for All Conference. ACM, New York, NY, USA, Article 3, 4 pages.

[13] Stephanie Ludi. 2007. Introducing accessibility requirements through external stakeholder utilization in an undergraduate requirements engineering course. In Proceedings of the 29th international conference on Software Engineering (ICSE '07). IEEE Computer Society, 736-743. DOI=10.1109/ICSE.2007.46

[14] Jennifer Mankoff. 2006. Practical service learning issues in $\mathrm{HCl}$. In Proceedings of CHI EA '06. ACM, New York, NY, USA, 201-206.

[15] MIT. 2015. 6.811: Principles and Practices of Assistive Technology (PPAT), Fall 2014. Retrieved April 28, 2017 from http://courses.csail.mit.edu/PPAT/fall2014/

[16] Geoff Norman. 2010. Likert scales, levels of measurement and the "laws" of statistics. Adv in Health Sci Educ (2010) 15:625-632. DOI 10.1007/s10459-010-9222$y$

[17] Helen L. Petrie and Alistair Edwards. 2006. Inclusive design and assistive technology as part of the $\mathrm{HCl}$ curriculum. In Proceedings of $\mathrm{HCl}$ Educators Workshop 2006. Pp. 23-24.

[18] Cynthia Putnam, Maria Dahman, Emma Rose, Jinghui Cheng, and Glenn Bradford. 2016. Best Practices for Teaching Accessibility in University Classrooms: Cultivating Awareness, Understanding, and Appreciation for Diverse Users. ACM Trans. Access. Comput. 8, 4, Article 13 (March 2016), 26 pages. DOI=http://dx.doi.org/10.1145/2831424

[19] Brian J. Rosmaita. 2006. Accessibility first! A new approach to web design. In Proceedings of the 37th SIGCSE technical symposium on Computer science education (SIGCSE '06). ACM, New York, NY, USA, 270-274. DOI=http://dx.doi.org/10.1145/1121341.1121426

[20] Shinohara, K., Bennett, C. L., and Wobbrock. J. O. (2016). How designing for people with and without disabilities shapes student design thinking. In Proceedings of the 9th international ACM SIGACCESS conference on Computers and accessibility (Assets '07). ACM, NY, USA, 123-130.

[21] Kathleen Tait and Nola Purdie. 2000. Attitudes toward disability: Teacher education for inclusive environments in an Australian university. International Journal of Disability, Development and Education, 47:1, 25-38.

[22] Teach Access. 2017. Retrieved April 28, 2017 from http://teachaccess.org

[23] Waller, A., Hanson, V.L., Sloan, D. 2009. Including accessibility within and beyond undergraduate computing courses. In Proceedings of the 11th international ACM SIGACCESS conference on Computers and accessibility (Assets '09). ACM, New York, NY, USA, 155-162. http://doi.acm.org/10.1145/1639642.1639670

[24] WebAIM. (2014). "Survey of Web Accessibility Practitioner Results." Retrieved April 28, 2017. http://webaim.org/projects/practitionersurvey/

[25] Jacob Cohen. 1960. A coefficient of agreement for nominal scales. Educational and Psychological Measurement, 20, 30-4

[26] Johnny Saldana. 2009. A coding manual for qualitative researchers. Sage.

[27] John L. Campbell, Charles Quincy, Jordan Osserman, and Ove K. Pedersen. 2013. "Coding In-depth Semistructured Interviews: Problems of Unitization and Intercoder Reliability and Agreement. Sociological Methods \& Research, 42, 3 (August 2013), 294-320. DOI: http://dx.doi.org/10.1177/0049124113500475 